\title{
Two-wavelength oximetry of tissue microcirculation based on sidestream dark-field imaging
}

Ryohei Hashimoto

Tomohiro Kurata

Masashi Sekine

Kazuya Nakano

Takashi Ohnishi

Hideaki Haneishi 


\title{
Two-wavelength oximetry of tissue microcirculation based on sidestream dark-field imaging
}

\author{
Ryohei Hashimoto, ${ }^{a}$ Tomohiro Kurata, ${ }^{b}$ Masashi Sekine, ${ }^{c}$ Kazuya Nakano, ${ }^{c, *}$ Takashi Ohnishi, ${ }^{c}$ and \\ Hideaki Haneishic $^{\mathrm{i}}$ \\ ${ }^{a}$ Chiba University, Graduate School of Science and Engineering, Chiba, Japan \\ 'Takano Co., Ltd., Nagano, Japan \\ ${ }^{\mathrm{c} C h i b a}$ University, Center for Frontier Medical Engineering, Chiba, Japan
}

\begin{abstract}
Monitoring oxygen saturation $\left(\mathrm{SO}_{2}\right)$ in microcirculation is effective for understanding disease dynamics. We have developed an $\mathrm{SO}_{2}$ estimation method, sidestream dark-field (SDF) oximetry, based on SDF imaging. SDF imaging is a noninvasive and clinically applicable technique to observe microcirculation. We report the first in vivo experiment observing the changes in $\mathrm{SO}_{2}$ of microcirculation using SDF oximetry. First, heat from the light-emitting diodes used for the SDF imaging might affect hemodynamics in microcirculation, hence, we performed an experiment to evaluate the influence of that on the SDF oximetry. The result suggested that SDF oximetry had enough stability for long-term experiments. Then, to evaluate the sensitivity of SDF oximetry to alterations in the hemodynamics of the microcirculation, we observed the time-lapsed $\mathrm{SO}_{2}$ changes in the dermis microcirculation of rats under hypoxic stimulation. We confirmed that the $\mathrm{SO}_{2}$ estimated by SDF oximetry was in accordance with changes in the fraction of inspired oxygen $\left(\mathrm{FiO}_{2}\right)$. Thus, SDF oximetry is considered to be able to observe $\mathrm{SO}_{2}$ changes that occur in accordance with alteration of the microcirculation. $\odot$ The Authors. Published by SPIE under a Creative Commons Attribution 3.0 Unported License. Distribution or reproduction of this work in whole or in part requires full attribution of the original publication, including its DOI. [DOI: 10.1117/1.JBO.24.3.031013]
\end{abstract}

Keywords: microcirculation; sidestream dark-field imaging; oximetry; hypoxia, oxygen saturation.

Paper 180397SSR received Jun. 29, 2018; accepted for publication Oct. 10, 2018; published online Oct. 30, 2018.

\section{Introduction}

Microcirculation is the blood flow through arterioles, capillaries, and venules, which exists throughout all tissues in a living body. Microcirculation plays an important role in supplying nutrients and oxygen to cells in the tissues. Thus, insufficient supplies of oxygen in microcirculation may lead to tissue hypoxia. Tissue hypoxia is considered to be one of the factors of serious diseases, such as cystic fibrosis and chronic bronchitis. ${ }^{1}$ Moreover, it is well known that varieties of experimental shock states (e.g., sepsis and haemorrhage) are related to hypoxia. ${ }^{2-4}$ In the early stages of these shock states, the impaired microcirculation will result in the rapid onset of tissue hypoxia. However, mechanisms of these diseases due to the hypoxia condition have not been clarified. Visualizing an individual microcirculation and observing the changes in the oxygen saturation $\left(\mathrm{SO}_{2}\right)$ during disease progression can be effective for understanding disease dynamics.

Many imaging techniques for $\mathrm{SO}_{2}$ in microcirculation have been developed. Snapshot multispectral imaging has been used for microcirculation oximetry mainly in the retina by using the difference in optical absorption properties of oxyhemoglobin $\left(\mathrm{HbO}_{2}\right)$ and deoxyhemoglobin (HbR). ${ }^{5}$ Photoacoustic imaging $(\mathrm{PAI})^{6}$ and optical coherence tomography $(\mathrm{OCT})^{7}$ also have been introduced to measure $\mathrm{SO}_{2}$ in microcirculation. However, these techniques require complex and expensive optical hardware. Therefore, simpler and lower cost optical setups would promote easier microcirculation assessment.

To visualize tissue microcirculation, orthogonal polarization spectral (OPS) imaging was developed as a real-time and

*Address all correspondence to: Kazuya Nakano, E-mail: knakano@ chiba-u.jp noninvasive imaging technique of handheld vital microscopes (HVMs) ${ }^{8}$ This imaging technique was used to reveal alterations of the microcirculation in patients with sepsis. ${ }^{9}$ The secondgeneration HVM is based on sidestream dark-field (SDF) imaging. ${ }^{10,11}$ This imaging technique can acquire higher contrast microcirculation images than OPS imaging. From the SDF images of individual vessels, some physical quantities such as vessel length, vessel diameter, and velocity of red blood cells (RBCs) can be estimated. ${ }^{12}$ The first commercial SDF device (Microscan, Microvision Medical B.V., Amsterdam, The Netherlands) has been developed. To verify its effectiveness, evaluation experiments on patients with diabetes were performed. ${ }^{11,13}$ The results showed that the microcirculation density of patients with diabetes was significantly higher than that of healthy people. Compared with other imaging techniques of microcirculation such as PAI and OCT, SDF imaging is a simple and low cost technique. Hence, SDF imaging is easily applicable to clinical purposes and enables users to observe the microcirculation of human tissues.

Based on SDF imaging, we have developed a trial SDF probe and spectral image-based oximetry method, which we called SDF oximetry. ${ }^{14,15}$ Our SDF oximetry utilizes two-wavelength oximetry based on the Beer-Lambert law. In another study, we applied average extinction coefficients (AECs) ${ }^{16}$ of hemoglobin to SDF oximetry and improved the accuracy of our method. However, in our SDF oximetry, there are three problems that have to be overcome. First, we have already confirmed the effectiveness of SDF oximetry in tissue-like turbid phantom experiments; however, we have not carried out the in vivo experiments. Second, since we calculate the AECs from the SDF images of the turbid phantom experiments, they are not optimized for in vivo SDF images. Third, heat from the light-emitting diodes (LEDs) used for the SDF imaging might affect hemodynamics 


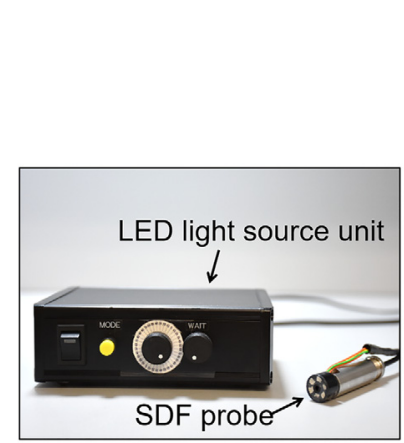

(a)

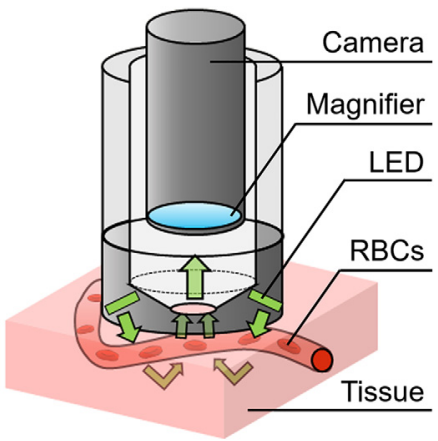

(b)
Fig. 1 Overview of our trial SDF imaging system. (a) Photo of the SDF imaging setup. (b) Schematic illustration of an image acquisition.

in microcirculation since the SDF probe is in direct contact with the tissue. We need to investigate the possible effect and that is the focus of this paper.

In this study, we conducted hypoxic stimulation experiments with rats by changing fraction of inspired oxygen $\left(\mathrm{FiO}_{2}\right)$. As a result, we confirmed that the estimated $\mathrm{SO}_{2}$ by SDF oximetry during the hypoxic stimulation experiment corresponded to the changes in $\mathrm{FiO}_{2}$. Prior to performing the in vivo experiment, we recalculated AECs from the literature values. Then, we examined the heat influence of the LEDs. Next, we conducted the experiment with rats where we observed the time-lapsed changes in $\mathrm{SO}_{2}$ under two conditions: normoxia and hypoxia. Then, we compared these $\mathrm{SO}_{2}$ values to evaluate our method, and the results suggested that SDF oximetry could response to alterations in microcirculation correctly.

\section{Materials and Methods}

\subsection{SDF Imaging}

Figure 1 shows the overview of our trial SDF imaging system. Our system comprises a light source unit and SDF probe, as shown in Fig. 1(a). Figure 1(b) is a schematic illustration of an image acquisition. The SDF probe is in direct contact with the tissue and the pulsed illumination from the LEDs placed concentrically around the probe directly penetrates into the tissue. SDF imaging can only capture the light diffused in the tissues without surface reflections because the camera inside the SDF probe is optically isolated from the peripheral illumination. In SDF images, blood vessels are represented in black due to optical absorption by hemoglobin molecules of the RBCs. As an imaging device, a complementary metal oxide semiconductor (CMOS) camera (ID04MB-IP-U, iDule Corporation, Chiba, Japan) with a customized magnifier (focal length: $5.6 \mathrm{~mm}$; $F$-number: F4.0) is used. Figure 2(a) shows the two-color LEDs (SMLVN6RGB1W, Rohm Co., Ltd., Kyoto, Japan) used in the system. The spectral intensities of the LEDs are shown in Fig. 2(b) together with extinction coefficients of $\mathrm{HbO}_{2}$ and $\mathrm{HbR}$ as a function of wavelength. ${ }^{17}$ The peak wavelengths of the two-color LEDs are $470 \mathrm{~nm}$ (blue) and $527 \mathrm{~nm}$ (green) and their full widths at half maximum are 27.3 and $37.2 \mathrm{~nm}$, respectively. The wavelength band of the blue LED is sensitive to changes in hemoglobin oxygenation, whereas that of the green LED is insensitive. Therefore, we can acquire microcirculation images in different contrasts corresponding to hemoglobin oxygenation.

\subsection{SDF Oximetry}

SDF oximetry utilizes two-wavelength oximetry based on the Beer-Lambert law. ${ }^{14}$ Figure 3 shows the assumption of $I_{\text {in }}(\lambda)$ and $I_{\text {out }}(\lambda)$ in the SDF imaging. We define the backreflection light entering into the blood vessel as the incident light $I_{\text {in }}(\lambda)$. When $I_{\text {in }}(\lambda)$ enters into the blood vessel with thickness $d$, the Beer-Lambert law gives the transmitted light $I_{\text {out }}(\lambda)$ through the medium as

$$
I_{\text {out }}(\lambda)=I_{\text {in }}(\lambda) \cdot \exp [-\varepsilon(\lambda) \cdot c \cdot d]
$$

where $\varepsilon(\lambda)$ represents the molar extinction coefficient of the hemoglobin and $c$ represents the total hemoglobin concentration. Hemoglobin has several variants, namely methemoglobin (MetHb), carboxyhemoglobin $(\mathrm{CoHb})$, and sulfhemoglobin (SulfHb). The absorption coefficients of these three hemoglobins have peaks in the range of visible light. ${ }^{18,19}$ However, concentrations of MetHb, $\mathrm{CoHb}$, and SulfHb included in blood are normally lower than $\mathrm{HbO}_{2}$ and $\mathrm{HbR}$, thus we can ignore them in

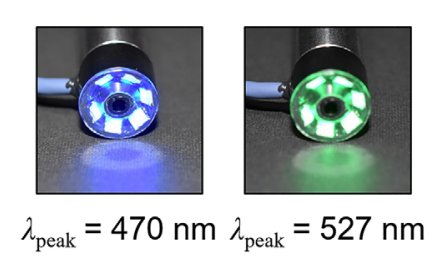

(a)

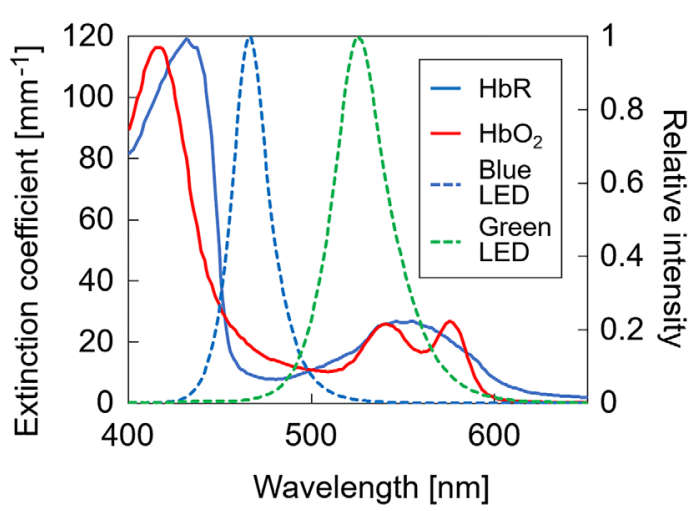

(b)

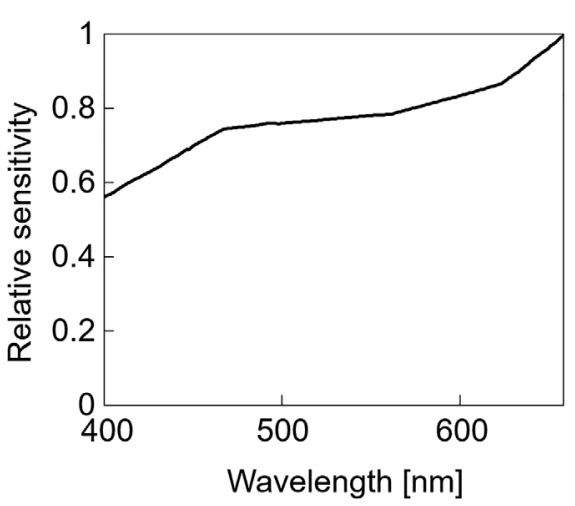

(c)

Fig. 2 The two-color LEDs used in the SDF imaging system. (a) Photos of LEDs emitting light. The colors are blue for the 470-nm peak wavelength and green for the 527-nm one. (b) Spectral distribution of extinction coefficients of $\mathrm{HbO}_{2}$ and $\mathrm{HbR}$ (left axis) and spectral intensity distribution of the two-color LEDs with the relative intensity (right axis). The intensities of blue and green LEDs at the peak wavelength are 27 and $4.5 \mathrm{~cd} / \mathrm{m}^{2}$, respectively. (c) Spectral sensitivity characteristics of the CMOS camera (relative value). 


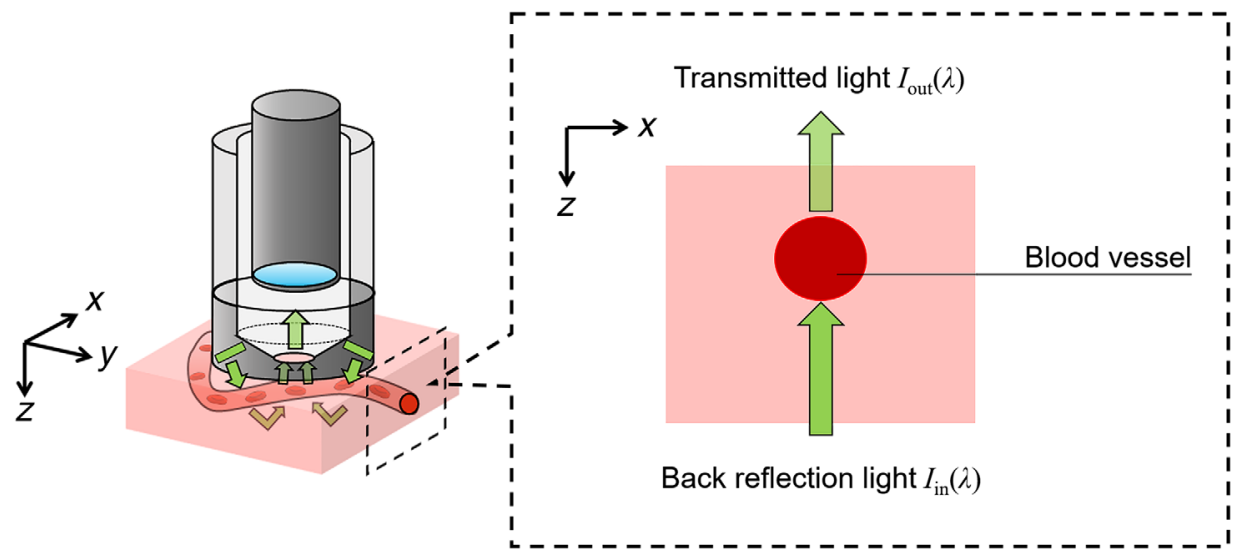

Fig. 3 Assumption of $I_{\text {in }}(\lambda)$ and $I_{\text {out }}(\lambda)$ in the SDF imaging.

our oximetry method. Moreover, absorption and scattering of leukocytes and platelets in blood occurs. ${ }^{20}$ However, these particles account for approximately only $4 \%$ of the whole blood volume, and hence they do not contribute more to absorption or scattering than RBCs do. As a result, for blood, $\varepsilon(\lambda)$ is approximately defined as

$\varepsilon(\lambda)=\mathrm{SO}_{2} \cdot \varepsilon_{\mathrm{HbO}_{2}}(\lambda)+\left(1-\mathrm{SO}_{2}\right) \varepsilon_{\mathrm{HbR}}(\lambda)$,

where the $\mathrm{SO}_{2}$ range is 0 to 1 . The optical density $\mathrm{OD}(\lambda)$ of blood is written as

$\mathrm{OD}(\lambda)=-\log \left[\frac{I_{\text {out }}(\lambda)}{I_{\text {in }}(\lambda)}\right]=\varepsilon(\lambda) \cdot c \cdot d$.

Finally, solving two equations of Eq. (3) for $\lambda_{1}$ and $\lambda_{2}$, we obtain $\mathrm{SO}_{2}$ as follows:

$\mathrm{SO}_{2}=\frac{\mathrm{OD}\left(\lambda_{2}\right) \cdot \varepsilon_{\mathrm{HbR}}\left(\lambda_{1}\right)-\mathrm{OD}\left(\lambda_{1}\right) \cdot \varepsilon_{\mathrm{HbR}}\left(\lambda_{2}\right)}{\mathrm{OD}\left(\lambda_{1}\right) \cdot \Delta \lambda_{2}-\mathrm{OD}\left(\lambda_{2}\right) \cdot \Delta \lambda_{1}}$,

where $\Delta \lambda_{n}=\varepsilon_{\mathrm{HbO} 2}\left(\lambda_{n}\right)-\varepsilon_{\mathrm{HbR}}\left(\lambda_{n}\right)$ for $n=1$,2. Figure 4 shows the definition of $I_{\text {in }}(\lambda)$ and $I_{\text {out }}(\lambda)$ in SDF images. In our definition, the intensity of vascular regions is treated as $I_{\text {out }}(\lambda)$ and that of avascular regions treated as $I_{\text {in }}(\lambda)$. Then, we can calculate $\mathrm{SO}_{2}$ by Eq. (4).

The $\mathrm{SO}_{2}$ value calculated by Eq. (4) cannot ignore the influence of the bandwidth of the LEDs shown in Fig. 2(b). We have to obtain the absorbance coefficients by wavelength averaging because the spectral distribution of the LEDs is not

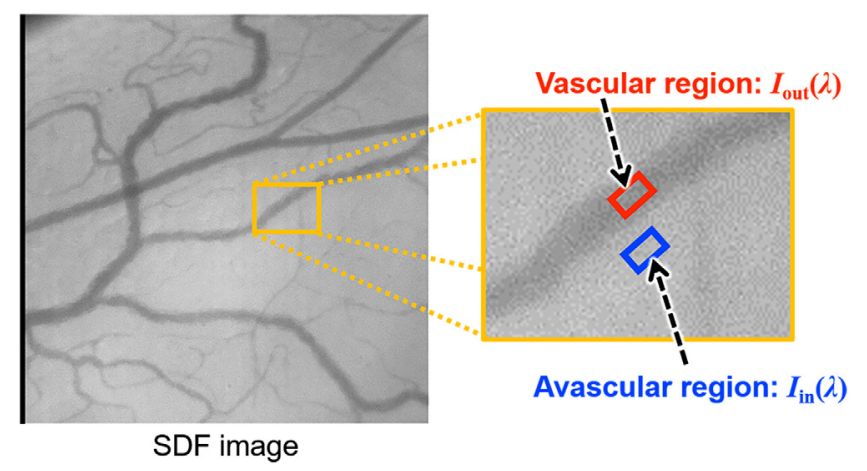

Fig. 4 Definition of $l_{\text {in }}(\lambda)$ and $l_{\text {out }}(\lambda)$ in the SDF image. truly monochromatic. To take into account the bandwidth of the LEDs, we apply AECs $\bar{\varepsilon}_{\text {blue }}$ and $\bar{\varepsilon}_{\text {green }}$ instead of $\varepsilon(\lambda){ }^{14}$ When the full width at 10th maximum is $w$, the intensity of light reaching the detector from vascular regions of $\mathrm{HbO}_{2}$ and $\mathrm{HbR}$ can be expressed as

$$
I_{\mathrm{out}, \mathrm{HbO}_{2}, k}=\int_{\lambda_{k}-\frac{1}{2} w}^{\lambda_{k}+\frac{1}{2} w} S(\lambda) \cdot L_{k}(\lambda) \cdot \exp \left[-\varepsilon_{\mathrm{HbO}_{2}}(\lambda) \cdot c \cdot d\right] \mathrm{d} \lambda,
$$

$I_{\mathrm{out}, \mathrm{HbR}, k}=\int_{\lambda_{k}-\frac{1}{2} w}^{\lambda_{k}+\frac{1}{2} w} S(\lambda) \cdot L_{k}(\lambda) \cdot \exp \left[-\varepsilon_{\mathrm{HbR}}(\lambda) \cdot c \cdot d\right] \mathrm{d} \lambda$,

where $S(\lambda)$ represents the spectral sensitivity of the camera and $L_{k}(\lambda)$ represents the spectral distribution of the $k^{\prime}$ th color of the LEDs. The intensity of light reaching the detector from avascular regions is expressed as

$I_{\mathrm{in}, k}=\int_{\lambda_{k}-\frac{1}{2} w}^{\lambda_{k}+\frac{1}{2} w} S(\lambda) \cdot L_{k}(\lambda) \mathrm{d} \lambda$

Hence, the AECs of $\mathrm{HbO}_{2}$ and $\mathrm{HbR}$ can be defined as

$$
\begin{aligned}
& \bar{\varepsilon}_{\mathrm{HbO}_{2}, k}=-\frac{1}{c d} \log \left(\frac{I_{\mathrm{out}, \mathrm{HbO}_{2}, k}}{I_{\mathrm{in}, k}}\right), \\
& \bar{\varepsilon}_{\mathrm{HbR}, k}=-\frac{1}{c d} \log \left(\frac{I_{\mathrm{out}, \mathrm{Hb}, k}}{I_{\mathrm{in}, k}}\right) .
\end{aligned}
$$

We calculated these AECs from the spectral distribution of the extinction coefficients of $\mathrm{HbO}_{2}$ and $\mathrm{HbR},{ }^{14}$ the spectral intensity distribution of the two-color LEDs, and the spectral sensitivity characteristics of the CMOS camera, shown in Figs. 2(b) and 2(c). The calculated results were $c d \bar{\varepsilon}_{\mathrm{HbO} \text {,blue }}=$ $13.5 \mathrm{~mm}^{-1}, c d \bar{\varepsilon}_{\mathrm{HbR}, \text { blue }}=8.47 \mathrm{~mm}^{-1}, c d \bar{\varepsilon}_{\mathrm{HbO} 2 \text {,green }}=11.2 \mathrm{~mm}^{-1}$, and $c d \bar{\varepsilon}_{\mathrm{HbR}, \text { green }}=11.2 \mathrm{~mm}^{-1}$.

\subsection{In Vivo Imaging Preparation}

Figure 5 is a photo of the experiment setup. In our experiment, we observed dermis microcirculation of male Slc:Wistar rats (260 to $280 \mathrm{~g}, 12$-weeks old, $n=3$ ). One was used for an 


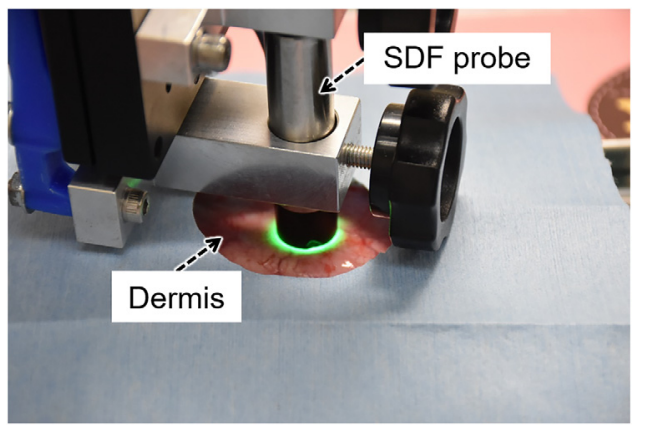

Fig. 5 In vivo experiment setup for SDF imaging.

experiment of stability evaluation, and the other two were for a hypoxic stimulation experiment. These rats were anesthetized under $2.0 \%$ isoflurane (Escain, Pfizer, Tokyo, Japan). A heat mat (Kainuma Industrial Co., Ltd., Aichi, Japan) was laid under the rat to help maintain its body temperature. To obtain clear images, the epidermis was removed before the observation. To monitor the percutaneous arterial oxygen saturation $\left(\mathrm{SpO}_{2}\right)$, a rat pulse oximeter (MouseOx Plus, STARR Life, Pennsylvania) was used.

As the first experiment, to ensure the time-lapsed changes in $\mathrm{SO}_{2}$ of hypoxic stimulation are not influenced by the heat from LEDs, a rat (rat 1) was observed during an 11-min period under normoxia. The rat's mouth and nose were covered with a mask that was connected to an inhalation tube. The SDF images were acquired under blue and green illuminations alternately every $100 \mathrm{~ms}$. Next, to assess the sensitivity of the SDF oximetry in accordance with physiological changes, 2 rats (rat 2 and 3) were exposed to hypoxic stimulation by changing $\mathrm{FiO}_{2}$. Similar procedures have been employed in previous studies. ${ }^{21,22}$ They were kept to inhale the air mixed with isoflurane for the first $3 \mathrm{~min}$. The oxygen concentration was then reduced to $10 \%$ oxygen by increasing nitrogen concentration together with isoflurane for $3 \mathrm{~min}$ to get the hypoxic stimulus state, which was followed by a switch back to the normal state for 5 min. The SDF images were acquired in the same manner as done in the first experiment. All experiments in this study were carried out in conformity with the Institutional Animal Care and Use policy of Chiba University.

\section{Experiments and Discussion}

\subsection{Time-Lapsed Stability Evaluation of SDF Oximetry}

The hypoxic stimulation experiment, described in Sec. 3.2, lasted $11 \mathrm{~min}$. Due to the long measurement time, there are two possible influences on the estimated $\mathrm{SO}_{2}$. The first is the heat influence caused by the LEDs. In SDF imaging, the SDF probe in which the LEDs are arranged is in direct contact with the tissue. Hence, the self-heating of LEDs caused by continuous light emission may affect microcirculation hemodynamics. The second is the influence of isoflurane. For example, Helmchen et al. ${ }^{23}$ observed anesthetic-induced changes in microcirculation hemodynamics. Therefore, we have to evaluate the stability of SDF oximetry during an 11-min period to ensure that time-lapsed changes in $\mathrm{SO}_{2}$ of hypoxic stimulation are not influenced by these two factors.

Prior to performing the in vivo experiment, we measured the time-lapsed changes in the temperature of the surface of

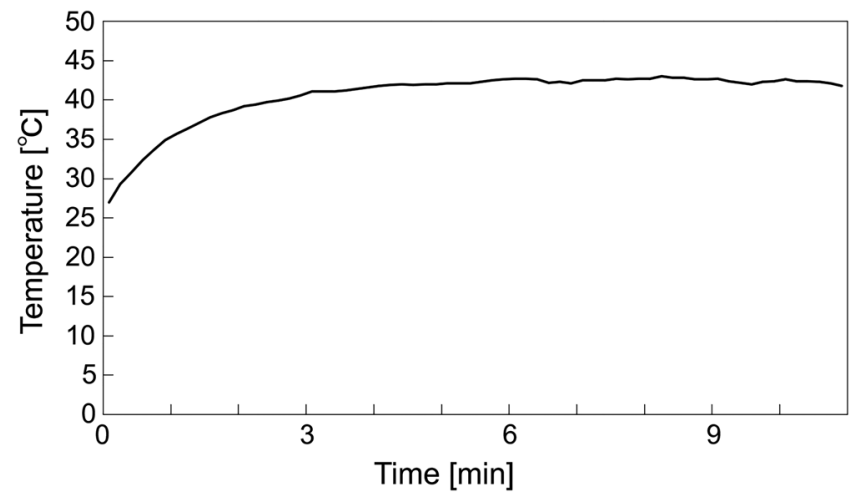

Fig. 6 Time-lapsed changes in the temperature of SDF probe surface.

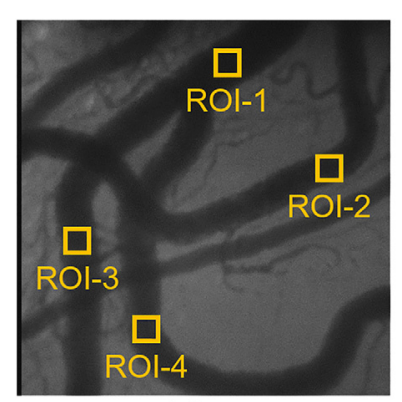

(a)

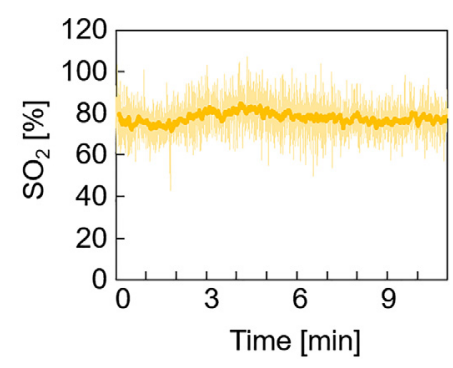

(b)
Fig. 7 Stability evaluation of our SDF oximetry. (Rat 1) (a) SDF image obtained under blue LED illumination at $0 \mathrm{~min}$. The labels ROI-1 to ROI-4 represent the regions of interest for $\mathrm{SO}_{2}$ estimation. (b) Time-lapsed changes in $\mathrm{SO}_{2}$ during $11 \mathrm{~min}$ for ROI-1. The orange solid line indicates the moving average for $6 \mathrm{~s}$.

SDF probe. We used a thermometer (AM-8051E, Anritsu Meter Co., Ltd., Tokyo, Japan) with a flexible internal temperature sensor (SF-E-200-ANP, Anritsu Meter Co., Ltd., Tokyo, Japan) for temperature measurement. As shown in Fig. 6, we found that the temperature of the SDF probe surface gradually increased and reached $\sim 42^{\circ} \mathrm{C}$. Then, we performed the in vivo experiment to evaluate the stability of SDF oximetry during an 11-min period. Figure 7(a) shows the SDF image obtained under the blue LED illumination at $0 \mathrm{~min}$. We analyzed the timelapsed changes in $\mathrm{SO}_{2}$ calculated by Eq. (4) for each region of interest (ROI) and calculated mean and standard deviation as shown in Fig. 7(b) and Table 1. The figure and table showed that there were no significant changes in $\mathrm{SO}_{2}$ during $11 \mathrm{~min}$. The results indicated the time-lapsed stability of our SDF oximetry. It means that in an 11 min-long experiment, there is no influence of self-heating of the LEDs and isoflurane. In Fig. 7(b), large variations in the estimated value of $\mathrm{SO}_{2}$ are observed. It is because that the two SDF images were acquired under blue and green illuminations alternately every $100 \mathrm{~ms}$. Since these

Table 1 Mean \pm standard deviation (\%) of estimated $\mathrm{SO}_{2}$ (rat 1 ).

\section{Region of interest}

\begin{tabular}{lccr}
\hline ROI-1 & ROI-2 & ROI-3 & ROI-4 \\
\hline $77.7 \pm 2.4$ & $90.0 \pm 4.8$ & $87.0 \pm 3.7$ & $82.6 \pm 5.6$ \\
\hline
\end{tabular}




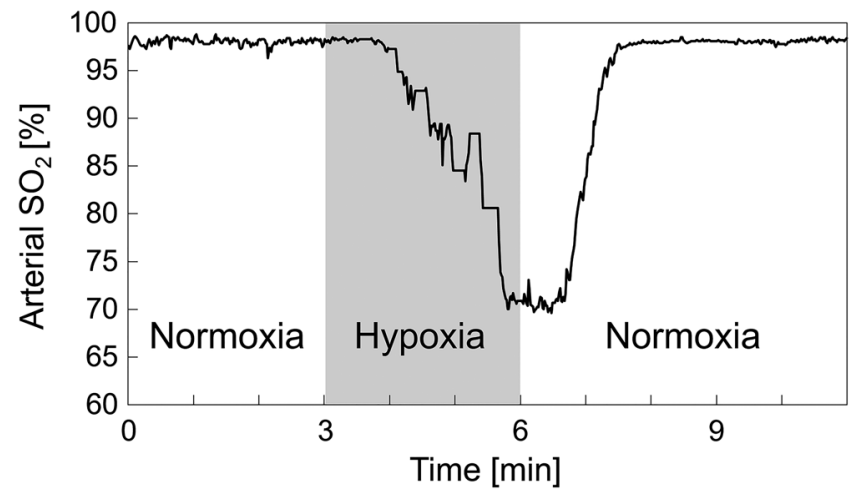

Fig. 8 Response of the $\mathrm{SpO}_{2}$ measured by the pulse oximeter during the experiment (rat 2).

images have a time-lag, each of their corresponding pixels used for $\mathrm{SO}_{2}$ estimation does not contain the exact same density of RBCs. If the density of RBCs within the pixel differs between two acquired SDF images, it results in an over- or underestimation of $\mathrm{SO}_{2}$. However, adopting a moving average, we can reduce the estimation error.

\subsection{Observation of Time-Lapsed Changes in $\mathrm{SO}_{2}$ During Hypoxic Stimulation}

The hypoxic stimulation experiment in this section was repeated using two rats. We describe results for rat 2 first. As shown in Fig. 8, the response of the $\mathrm{SpO}_{2}$ measured by the pulse oximeter during the hypoxic stimulation experiment corresponded to the change in $\mathrm{FiO}_{2}$. The decrease and increase in $\mathrm{SpO}_{2}$ started $\sim 1$ min after the change in $\mathrm{FiO}_{2}$. Figure 9 shows the timelapsed SDF images. We could observe a slight vasoconstriction and a decrease in blood flow in accordance with the hypoxic stimulation. The vasoconstriction is caused by temporary elevation of blood pressure associated with hypoxia. ${ }^{24}$ After hypoxia, these two characteristics returned to their original state. In the $\mathrm{SO}_{2}$ analysis, we focused on two blood vessels. One had a bifurcation in the field of view (blue arrow) and the other had no bifurcation (red arrow). In particular, we predicted that the blood vessel indicated by the blue arrow was the venule because the blood flow was merged at the vessel branch.

$\mathrm{SO}_{2}$ of individual vessels were calculated for each pixel from two-band SDF images. Figure 10 shows the changes in $\mathrm{SO}_{2}$ maps during hypoxic stimulation. The results clearly showed that the $\mathrm{SO}_{2}$ in microcirculation decreased during hypoxic stimulation and increased after hypoxia. Figure 11(b) shows the changes in estimated $\mathrm{SO}_{2}$ during hypoxic stimulation corresponding to the rectangular area shown in Fig. 11(a). The size of the area was set to $20 \times 20$ pixels. The gray-shaded area meant the period of hypoxic stimulation. These results mostly corresponded to the change in the $\mathrm{SpO}_{2}$, measured by the pulse oximeter shown in Fig. 8. After returning to the normoxia stimulation, the estimated $\mathrm{SO}_{2}$ increased nearly $100 \%$ almost instantaneously and then decreased gradually. This result might reflect the function of microcirculation to regulate the tissue oxygenation.

Further, we focused on the difference in response to hypoxia between two blood vessels, which are marked by the red and blue arrows in Fig. 11. As shown in Fig. 11(b), the $\mathrm{SO}_{2}$ of the blue rectangular area began to change before there were observable changes in the red rectangular area. These differences between the two blood vessels may be due to the difference between arterioles and venules as mentioned in a previous study. ${ }^{5}$ In SDF imaging, arterioles and venules are distinguished by the blood flow direction at the vessel branch. However, more accurate identification requires a larger image size than that of the present $\mathrm{SDF}$ images $\left(0.8 \times 0.8 \mathrm{~mm}^{2}\right)$.

In order to ensure the reproducibility of the hypoxic stimulation experiment, we repeated it with the other rat (rat 3). The experimental conditions were the same as the first time. Figure 12 shows that the created $\mathrm{SO}_{2}$ maps had a tendency

\section{Before hypoxia}
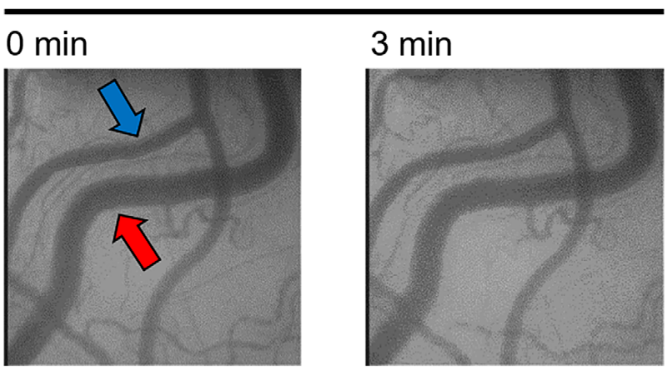

\section{During hypoxia}

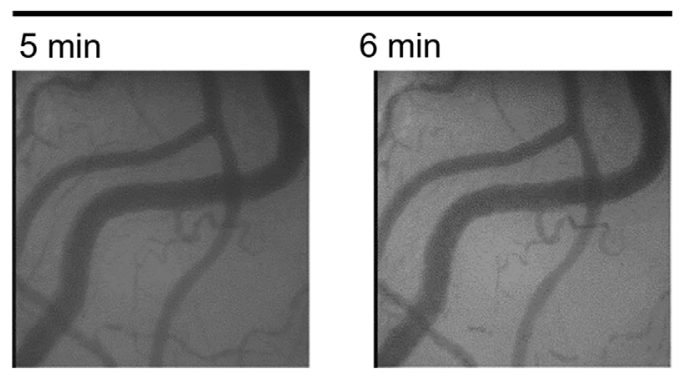

After hypoxia

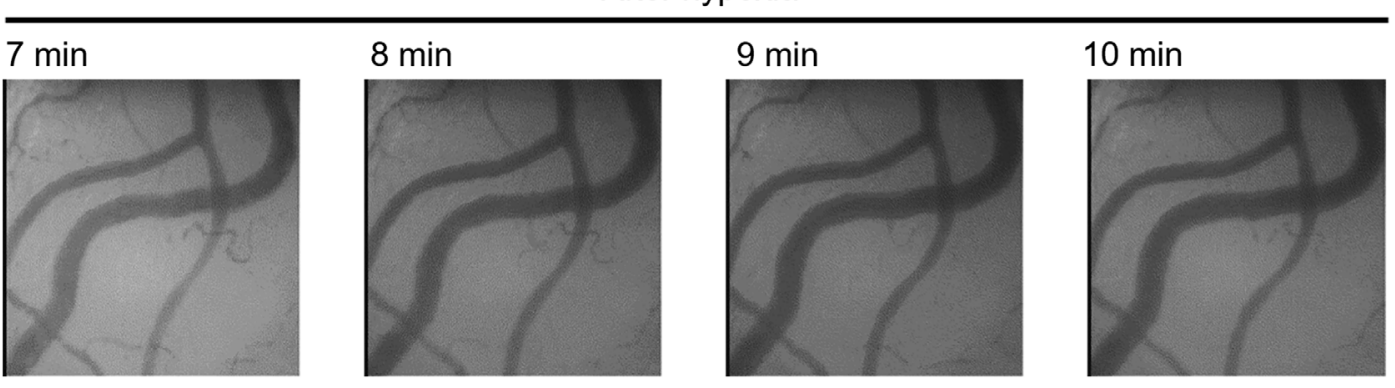

Fig. 9 Time-lapsed SDF images during hypoxic stimulation experiment (rat 2). 


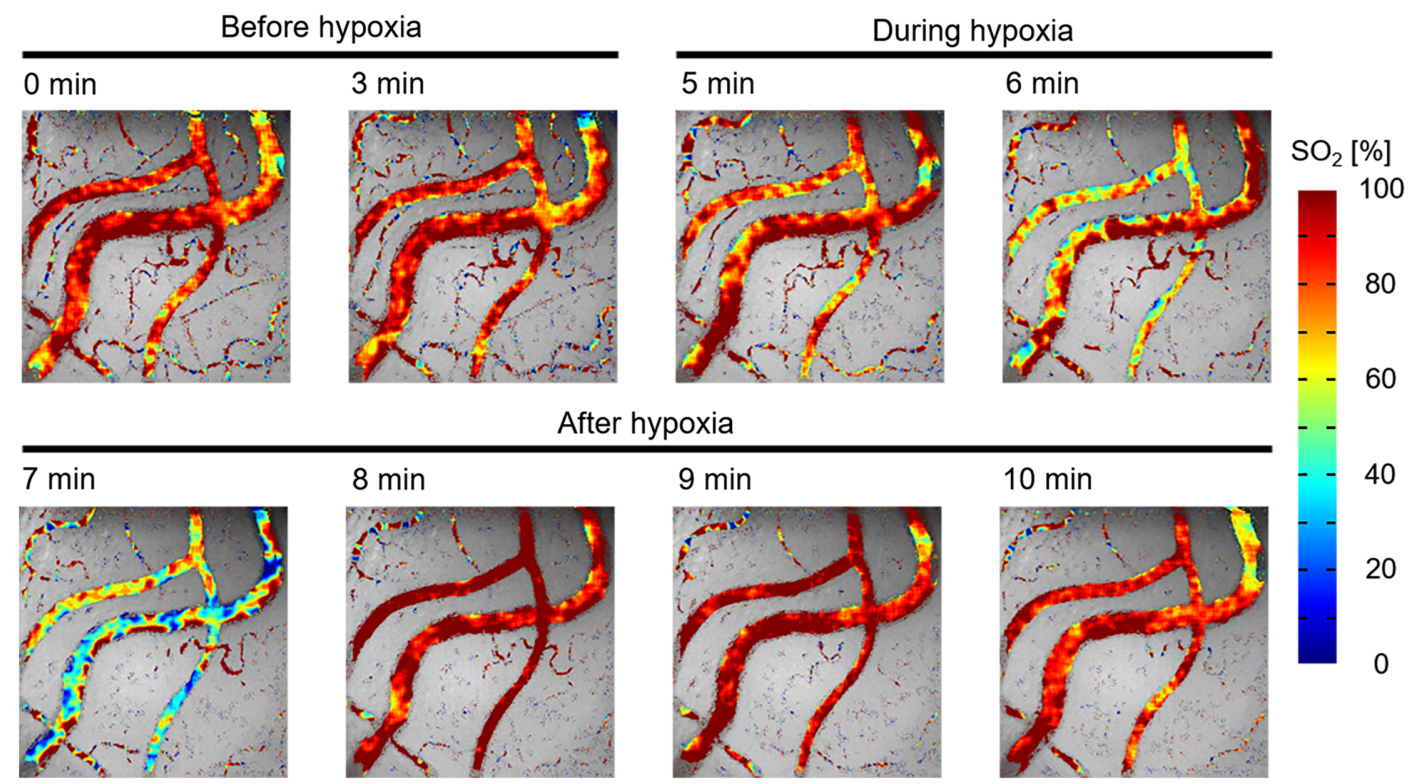

Fig. 10 Alterations of $\mathrm{SO}_{2}$ maps during hypoxic stimulation (rat 2).

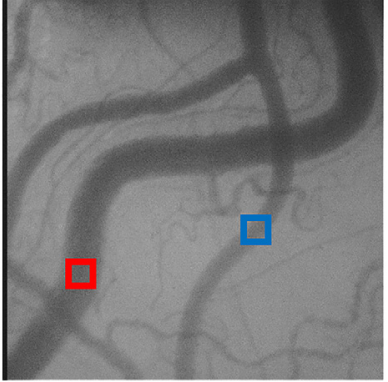

(a)

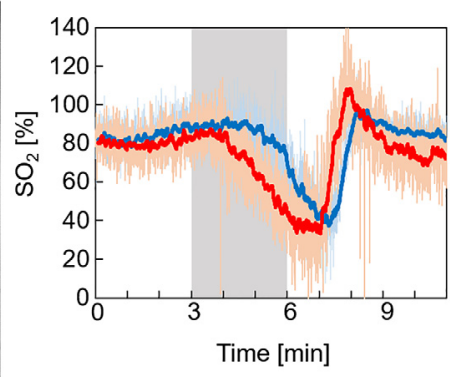

(b)
Fig. 11 Changes in estimated $\mathrm{SO}_{2}$ during hypoxic stimulation (rat 2). (a) Obtained SDF image. (b) Time-lapsed changes in $\mathrm{SO}_{2}$ during hypoxic stimulation. The red and blue solid lines indicate the moving average for $6 \mathrm{~s}$. similar to that of Fig. 10. In addition, the difference in the change of timing between different vessels was also observed as shown in Fig. 13. In the repeated experiment, the response speed for the estimated $\mathrm{SO}_{2}$ to hypoxia was faster than that of the first. We considered that to be due to individual differences between the rats or to differences of the blood vessel diameters. Figure 14 shows SDF images obtained at 0 and $7 \mathrm{~min}$. There were two characteristics. First, the blood vessel indicated by the red arrow decreased in diameter compared with that indicated by the blue arrow. To compensate for the insufficient oxygen supply, the diameter of the arterioles presumably varied for the increasing blood pressure. Second, blood vessel density in the areas indicated by the yellow circles was reduced by hypoxia. We considered that the decrease in blood flow caused by hypoxia resulted in the lack of perfusion to capillaries and the reduction of blood vessel density.
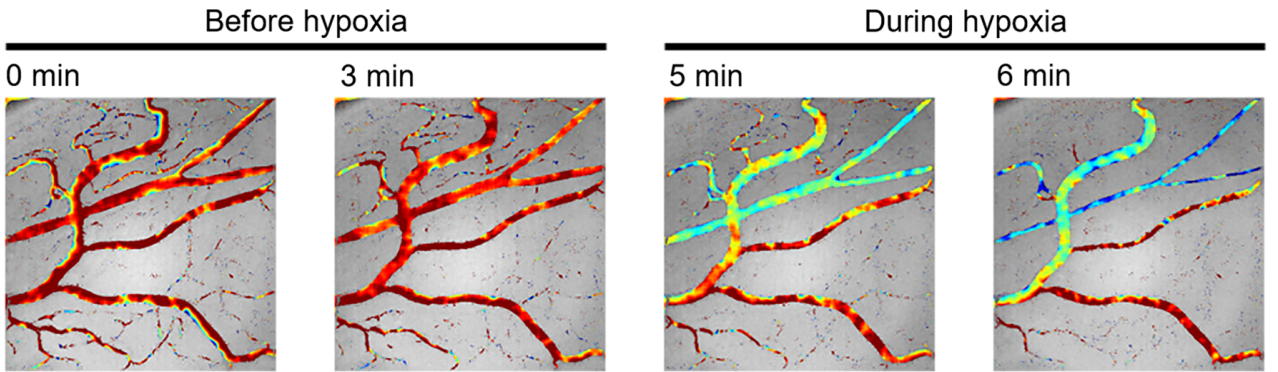

$\mathrm{SO}_{2}[\%]$
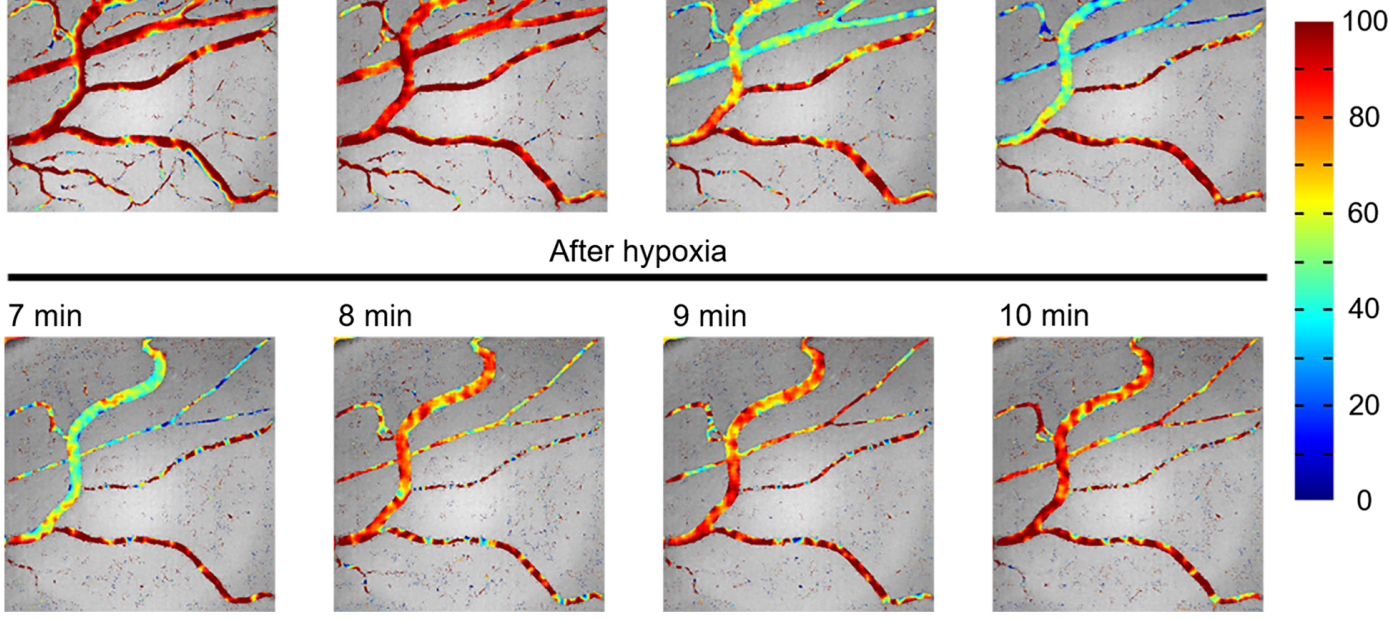

After hypoxia
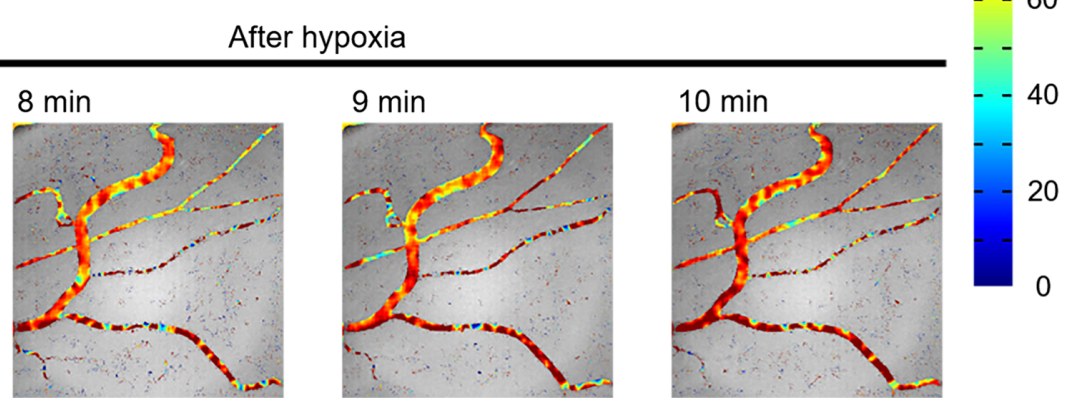

Fig. 12 Alterations of $\mathrm{SO}_{2}$ maps during hypoxic stimulation (rat 3). 


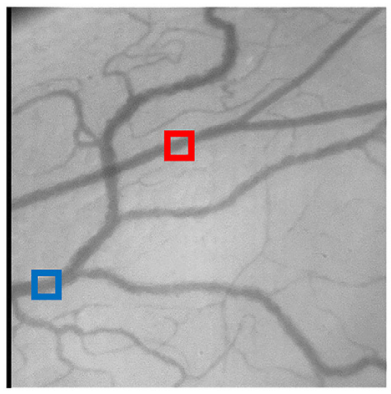

(a)

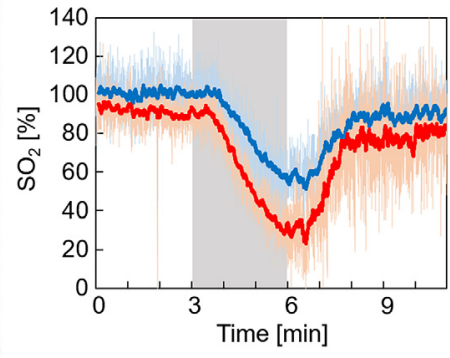

(b)
Fig. 13 Changes in estimated $\mathrm{SO}_{2}$ during hypoxic stimulation (rat 3). (a) Obtained SDF image. (b) Time-lapsed changes in $\mathrm{SO}_{2}$ during hypoxic stimulation. The red and blue solid lines indicate the moving average for $6 \mathrm{~s}$.

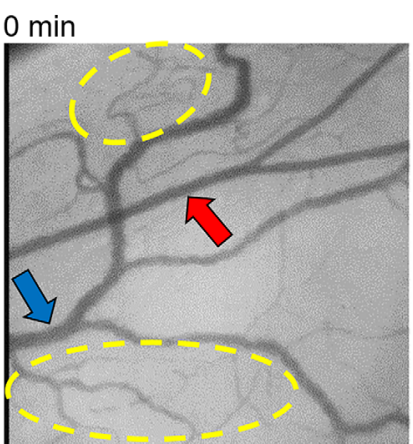

(a)

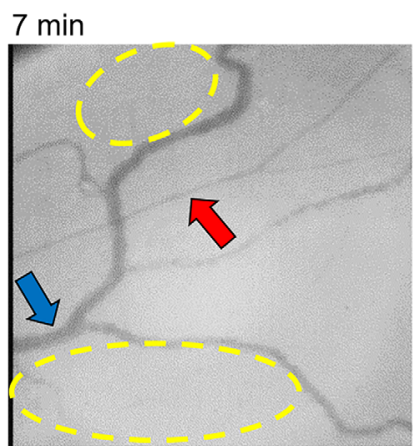

(b)
Fig. 14 Comparison of blood vessel structure between before and after hypoxia (rat 3).

Our results showed that SDF oximetry could estimate $\mathrm{SO}_{2}$ of microcirculation and respond to an alteration in microcirculation in hypoxic stimulation experiments with rats. This indicates that SDF oximetry can be applied for understanding microcirculatory dysfunction in oxygen delivery induced by several diseases, as mentioned in the introduction. However, the accuracy of absolute $\mathrm{SO}_{2}$ value estimated by SDF oximetry is uncertain. Therefore, we have to compare the $\mathrm{SO}_{2}$ value estimated by SDF oximetry with the one obtained by using high accuracy oximeter (e.g., blood gas analyzer).

\section{Conclusion}

In this paper, we have applied our SDF oximetry to in vivo experiments and observed the time-lapsed $\mathrm{SO}_{2}$ changes in dermis microcirculation of rats under hypoxic stimulation. Before performing the in vivo experiment, we calculated the AECs from the literature values because the AECs used in our previous study were optimized only for turbid phantom experiments. Next, to confirm the time-lapsed stability of SDF oximetry, we obtained SDF images under the normoxia during an 11min period. The results suggested that hemodynamics in microcirculation were not affected by the heat from the LEDs. Then, we conducted the in vivo experiment with rats to evaluate the sensitivity of SDF oximetry under hypoxic stimulation. This is the first in vivo experiment that observed the changes in $\mathrm{SO}_{2}$ of microcirculation based on SDF imaging.

We confirmed that the estimated $\mathrm{SO}_{2}$ by SDF oximetry was in accordance with the change in $\mathrm{FiO}_{2}$. Therefore, SDF oximetry is considered to be a method that enables us to observe $\mathrm{SO}_{2}$ changes occurring in accordance with alteration of microcirculation. Moreover, we focused on not only the overall $\mathrm{SO}_{2}$ changes in microcirculation but also the difference between individual blood vessels. The speeds of these responses to hypoxic stimulation were different. These differences were thought to be due to the difference between arterioles and venules as reported previously, ${ }^{5}$ or to differences of blood vessel diameter.

To raise the reliability of $\mathrm{SO}_{2}$ estimations, future work must take into account the error factors of SDF oximetry such as the influence of scattering by tissues, as mentioned in our previous study. ${ }^{15}$ As one solution to this problem, Monte Carlo simulation of photon propagation is considered. Analyzing the photon propagation in the tissue would enable researchers to understand the behavior of photons and lessen the extent of the error factors. After improving the accuracy of $\mathrm{SO}_{2}$ estimation, as future work, we will conduct in vivo experiments with a sepsis rat model by following the same procedure as described in our previous work. ${ }^{25}$ Then, we will investigate the relationship between oxygen delivery and mechanism of sepsis shock.

\section{Disclosures}

All authors have no conflicts of interest or financial ties to disclose.

\section{Acknowledgments}

This research was supported by KAKENHI (the Grants-in-aid for Scientific Research) (A) 16H01855.

\section{References}

1. R. E. Airley, J. E. Monaghan, and I. J. Stratford, "Hypoxia and disease: opportunities for novel diagnostic and therapeutic prodrug strategies," Pharm. J. 264(7094), 666-673 (2000).

2. C. G. Ellis, J. Jagger, and M. Sharpe, "The microcirculation as a functional system," Crit. Care 9(4), S3 (2005).

3. C. Ince and M. Sinaasappel, "Microcirculatory oxygenation and shunting in sepsis and shock," Crit. Care Med. 27(7), 1369-1377 (1999).

4. J. W. Cannon, "Hemorrhagic shock," N. Engl. J. Med. 378(4), 370-379 (2018).

5. H. C. Hendargo et al., "Snap-shot multispectral imaging of vascular dynamics in a mouse window-chamber model," Opt. Lett. 40(14), 3292-3295 (2015)

6. S. Hu and L. V. Wang, "Photoacoustic imaging and characterization of the microvasculature," J. Biomed. Opt. 15(1), 011101 (2010).

7. S. P. Chong et al., "Quantitative microvascular hemoglobin mapping using visible light spectroscopic optical coherence tomography," Biomed. Opt. Express 6(4), 1429-1450 (2015).

8. W. Groner et al., "Orthogonal polarization spectral imaging: a new method for study of the microcirculation," Nat. Med. 5(10), 1209-1212 (1999).

9. D. De Backer et al., "Microvascular blood flow is altered in patients with sepsis," Am. J. Respir. Crit. Care Med. 166(1), 98-104 (2002).

10. C. Ince, "Sidestream dark field imaging: an improved technique to observe sublingual microcirculation," Crit. Care 9(1), P72 (2005).

11. P. T. Goedhart et al., "Sidestream dark field (SDF) imaging: a novel stroboscopic LED ring-based imaging modality for clinical assessment of the microcirculation," Opt. Express 15(23), 15101-15114 (2007).

12. J. G. Dobbe et al., "Measurement of functional microcirculatory geometry and velocity distributions using automated image analysis," Med. Biol. Eng. Comput. 46(7), 659-670 (2015).

13. R. Djaberi et al., "Non-invasive assessment of microcirculation by sidestream dark field imaging as a marker of coronary artery disease in diabetes," Diabetes Vasc. Dis. Res. 10(2), 123-134 (2013). 
14. T. Kurata et al., "Impact of vessel diameter and bandwidth of illumination in sidestream dark-field oximetry," Biomed. Opt. Express 6(5), 1616-1631 (2015).

15. T. Kurata et al., "Correction method for influence of tissue scattering for sidestream dark-field oximetry using multicolor LEDs," Opt. Rev. 23(6), 955-967 (2016).

16. A. J. Cohen and R. A. Laing, "Multiple scattering analysis of retinal blood oximetry," IEEE Trans. Biomed. Eng. BME-23, 391-400 (1976).

17. D. J. Faber et al., "Oxygen saturation-dependent absorption and scattering of blood," Phys. Rev. Lett. 93(2), 028102 (2004).

18. W. G. Zijlstra et al., "Spectrophotometry of hemoglobin: absorption spectra of bovine oxyhemoglobin, deoxyhemoglobin, carboxyhemoglobin, and methemoglobin," Comp. Biochem. Physiol. B: Biochem. Mol. Biol. 118(4), 743-749 (1997).

19. A. Zwart et al., "A multi-wavelength spectrophotometric method for the simultaneous determination of five haemoglobin derivatives," Clin. Chem. Lab. Med. 19(7), 457-464 (1981).

20. M. C. Meinke et al., "Optical properties of platelets and blood plasma and their influence on the optical behavior of whole blood in the visible to near-infrared wavelength range," J. Biomed. Opt. 12(1), 014024 (2007).

21. L. E. MacKenzie et al., "In vivo oximetry of human bulbar conjunctival and episcleral microvasculature using snapshot multispectral imaging," Exp. Eye Res. 149, 48-58 (2016).

22. W. Feng et al., "Lookup-table-based inverse model for mapping oxygen concentration of cutaneous microvessels using hyperspectral imaging," Opt. Express 25(4), 3481-3495 (2017).

23. F. Helmchen et al., "A miniature head-mounted two-photon microscope: high-resolution brain imaging in freely moving animals," Neuron 31(6), 903-912 (2001).

24. M. Sugimura et al., "Influence of acute progressive hypoxia on cardiovascular variability in conscious spontaneously hypertensive rats," Auton. Neurosci. Basic Clin. 141(1), 94-103 (2008).

25. M. Takahashi et al., "Quantitative evaluation of blood flow obstruction in microcirculation with sidestream dark-field images," Proc. SPIE 10068, 100680A (2017).
Ryohei Hashimoto is an MS student at the Graduate School of Science and Engineering, Chiba University, Japan, where he received his BS degree in engineering in 2017.

Tomohiro Kurata received his MS degree from Gakushuin University in 2012 and his PhD from Chiba University in 2017. He is an R\&D engineer at Takano Co., Ltd. His current research interests include image processing, diffuse reflectance spectroscopy, and multispectral imaging.

Masashi Sekine received his PhD from Chiba University in 2015. He is a chief technical officer at the Center for Frontier Medical Engineering, Chiba University, and a part-time lecturer at the College of Engineering and Design, Shibaura Institute of Technology. His research interests include mechanical design, robotics, production engineering, and medical engineering. Currently, he is engaged in developing rehabilitation devices including prostheses.

Kazuya Nakano received his MS and PhD degrees from Tokyo Institute of Technology in 2007 and 2013, respectively. He is a specially appointed assistant professor at the Center for Frontier Medical Engineering, Chiba University. His current research interests include diffuse reflectance spectroscopy, multispectral imaging, optical security, information, and photonics.

Takashi Ohnishi received his MS and PhD degrees from Chiba University in 2010 and 2013, respectively. He is an assistant professor at the Center for Frontier Medical Engineering, Chiba University. His current research interests include image processing, image registration, and pathological images.

Hideaki Haneishi received his MS degree in 1987 and his PhD in 1990 from Tokyo Institute of Technology. He joined Chiba University in 1990 as a research associate. He was a visiting research scientist at the Department of Radiology, University of Arizona, from 1995 to 1996 . He has been a full professor at the Center for Frontier Medical Engineering (CFME) since 2007. Currently, he is also a director of CFME. 